\title{
Avaliação da infecção por helmintos gastrintestinais em caprinos criados na região metropolitana do Recife - PE através de exame coproparasitológico e larva cultura
}

\section{Avaluation of the infection for gastrointestinal helminths in goats from the metropolitan area of Recife - Pernambuco state - Brasil through the faecal egg count and larval culture}

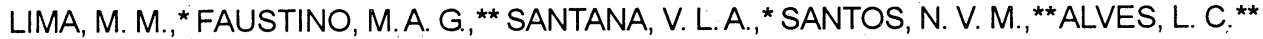

\begin{abstract}
Resumo
A caprinocultura é uma atividade de relevância social e econômica para o país, alcançando expansão significativa em várias regiōes brasileiras, principalmente na região Nordeste. Além dos problemas com manejo zootécnico e sanitário enfrentados pela caprinocultura brasileira, somam-se condições climáticas que favorecem o desenvolvimento de diferentes parasitoses, principalmente as helmintoses, sendo consideradas um entrave à expansão da caprinocultura no Brasil. Este trabalho teve como objetivo estudar as infecções por helmintos gastrintestinais em caprinos criados na região metropolitana do Recife através da contagem de ovos por grama de fezes (OPG) e identificação de larvas de terceiro estágio obtidas em coproculturas. Foram utilizados em média 20 caprinos de uma propriedade localizada no município de Paulista dos quais, mensalmente, em datas preestabelecidas, coletaram-se amostras de fezes individuais no período de agosto de 1998 a julho de 1999 . Observouse um percentual de positividade de $82 \%$. Das amostras examinadas, $72,8 \%$ apresentavam ovos tipo Strongyloidea, $8,4 \%$ Moniezia spp. e $2 \%$ Trichuris sp. Nas coproculturas foram identificadas larvas de terceiro estágio de Haemonchus sp. $(75,13 \%)$, Trichostrongylus spp. $(24,32 \%)$ e Oesophagostomum spp. (0,54\%). O número médio de larvas por grama de fezes para os gêneros Haemonchus e Trichostrongylus foi maior nos meses de maior precipitação pluviométrica. Obteve-se correlação estatisticamente significativa entre OPG e temperatura, OPG e precipitação pluviométrica, e entre OPG e o número de larvas de Haemonchus sp. por grama de fezes. Haemonchus sp. ocorreu em todos os meses do período em estudo.
\end{abstract}

Palavras-Chave: caprino, helmintos, OPG, coprocultura.

\begin{abstract}
The goat breeding is an activity of social and economical relevance to the Country, reaching significant expansion in several Brazilian areas, mainly in the Northeast. Besides the problems with sanitary handling, the Brazilian climatic conditions favour the development of different parasitosis, between them, the helmintic infections are considered as a fetter to the expansion of this activity in Brazil. This work had as objective to study the infections for gastrointestinal helminths in goat breed in the Metropolitan Area of Recife - Perriambuco state - Brazil through the count of eggs per gram of faeces (EPG) and identification of the third stage of larvae of nematode. There were used an average of 20 animals from a property located in the municipal district of Paulista. Of these animals, monthly, in pre-established dates individual faecal samples were collected in the period of August of 1998 to July of 1999 . It was observed $82 \%$ of positivity., Strongyloidea type eggs was observed in $72.8 \%$ of the samples, $8.4 \%$ presented Moniezia spp, and $2 \%$ Trichuris sp. Third stage larvae of Haemonchus sp. were present in $75.13 \%$, Trichostrongylus spp. in $24,32 \%$ and Oesophagostomum spp. in $0,54 \%$. The medium number of larvae per gram of faeces for the genus Haemonchus and Trichostrongylus was larger in the months with higher rainfall. There was significant correlation among EPG. and temperature, EPG and rainfall, and between EPG and the number of larvae of Haemonchus sp. per gram of faeces. Haemonchus sp. was present in all months of the period.
\end{abstract}

Keywords: goats, helminths, EPG.

\section{Introdução}

A caprinocultura representa uma das principais fontes de proteina para alimentação humana, o que torna esta exploração uma atividade de relevante importância socioeconômica. En- tretanto, a produtividade destes animais é comprometida devido a problemas de ordem sanitária, nutricional e de manejo (Vieira, 1999). Além dos problemas com manejo zootécnico e sanitário enfrentados pela caprinocultura brasileira, somamse condições climáticas que favorecem o desenvolvimento

\footnotetext{
* Médica-veterinária - MSc. Doutoranda (PPGCV - UFRPE) R.Bernardo Sayão, 84, Várzea, CEP 50741-440 - Recife, PE
}

** Prof. DMVIUfRPE, Rua Dom Manoel de Medeiros, s/n Dois Irmãos CEP 50000 - Recife, PE 
de diferentes parasitoses (Santos, 1982), principalmente as helmintoses, sendo consideradas um entrave à expansão da caprinocultura no país, responsáveis por elevadas perdas econômicas, em decorrência de crescimento retardado, perda de peso, redução no consumo de alimentos, queda na produção de leite, baixa fertilidade e, nos casos de infecções maciças, altas taxas de mortalidade em caprinos (Vieira, 1999).

O Brasil está colocado entre os dez países possuidores dos maiores rebanhos caprinos do mundo (Castro, 1981; Cunha, 1982), contando com um efetivo de 8.164.153 (IBGE, 1998). A maior população desta espécie está concentrada no Nordeste, sendo 7.596 .256 cabeças, o que corresponde a $90,03 \%$ do rebanho nacional. O rebanho caprino de Pernambuco é constituido por 1.239.331 cabeças (IBGE,1998), destacandose os animais sem raça definida (SRD), os quais se constituem maioria no rebanho caprino do país.

Costa (1980) relaciona os helmintos parasitas de caprinos no Nordeste do Brasil, referindo-se às espécies $H$. contortus, Trichostrongylus colubriformis, Strongyloides e Oesophagostomum columbianum como as mais prevalentes. Charles (1989), na cidade de Petrolina em Pernambuco, verificou, em caprinos não tratados, um maior número de helmintos durante o final da época chuvosa e início da época seca (março/junho) e um número menor no meio da época chuvosa (janeiro/fevereiro), sendo mais prevalentes as espécies $H$. contortus, $S$. papillosus e O. columbianum. De acordo com Pinheiro (1983), nas zonas de verão chuvoso as espécies de helmintos principais são Haemonchus contortus e Oesophagostomum columbianum; nas zonas de inverno chuvoso, os helmintos de maior significado parasitológico são Ostertagia circuncincta, Trichostrongylus axei, T. vitrinus e T. Colubriformis e nas zonas de chuvas não definidas os parasitas de maior importância econômica são Ostertagia sp. e Trichostrongylus $s p$.

Girão et al. (1992), no Piauí, observaram que a maior quantidade de ovos por gramas de fezes (OPG) dos caprinos traçadores ocorreu durante e após o periodo chuvoso apresentando pequena quantidade nos meses de agosto, setembro e outubro (período seco) enquanto nos caprinos testemunhas, o OPG apresentou-se elevado no final da época chuvosa e durante o período seco. Estudos recentes de Morales et al. (1998) consideram que a contagem do número de ovos por grama de fezes implica o reconhecimento de uma relação entre o número de parasitas presentes no trato gastrintestinal e o número de ovos eliminados com as fezes, o que justifica o amplo uso deste método.

$\mathrm{Na}$ região Nordeste existem poucos trabalhos que avaliem a infecção helmíntica em caprinos. Sabendo-se que numerosos fatores interagem no ecossistema, provocando grande variação, na dinâmica populacional do helminto, mesmo em regiões próximas (Costa et al., 1970, 1971, 1973; Santiago, 1979), desenvolveu-se este trabalho com objetivo de se obter dados referentes à infecção por parasitos gastrintestinais em caprinos criados na região metropolitana do Recife, PE, através de exames coproparasitológicos e larva cultura.

\section{Material e métodos}

O estudo foi conduzido em uma propriedade de criação de caprinos localizada no municipio de Paulista - PE (Latitude Leste 07056'27", Longitude Oeste 3452'23"). Utilizaram-se, em média, mensalmente, 20 caprinos das raças Saanen e mestiços (Saanen e Pardo Alpina), machos e fêmeas, idades variadas, criados em sistema semi-intensivo, alimentados com capim, concentrado, ${ }^{1}$ sal mineral e água ad libitum, dos quais, mensalmente, em datas preestabelecidas, no periodo de agosto de 1998 a julho de 1999, coletaram-se amostras de fezes.

O esquema anti-helmíntico nesta propriedade foi realizado com produto comercial à base de ivermectina, utilizado conforme indicações do fabricante. As vermifugações se concentraram nos meses de menor precipitação pluviométrica, respeitando-se o esquema adotado pelo proprietário, sendo reduzidas nos meses do periodo chuvoso.

Os dados meteorológicos dos referidos municípios foram obtidos no Instituto Nacional de Meteorologia - 3 Distrito de Meteorologia da cidade de Recife, PE.

\section{Coleta do material}

A coleta de fezes foi realizada diretamente do reto dos animais, utilizando-se sacos plásticos identificados com dados do animal, da propriedade e data da coleta. Em seguida, as amostras foram acondicionadas em caixa isotérmica contendo gelo reciciável, para transporte até o Laboratório de Doenças Parasitárias dos Animais Domésticos - Departamento de Medicina Veterinária - UFRPE.

\section{Procedimentos laboratoriais}

As amostras fecais foram individualmente processadas para a contagem de ovos por grama de fezes (OPG) segundo Gordon e Whitłock (1939). Sendo o grau de infecção interpretado segundo Ueno e Gonçalves (1998). Paralelamente, realizou-se a identificação e contagem de larvas de terceiro estágio de nematóides gastrintestinais por grama de fezes, através de coprocultura pela técnica de Roberts e O'Sullivan (1950).

\section{Análise estatística}

O estudo da infecção foi analisado através da média e desvio-padrão do OPG e LPG obtidos mensalmente para cada parasito identificado.

A análise de correlação, através do cálculo do coeficiente de correlação de Pearson, foi utilizada para determinação do grau de associação entre as variáveis meteorológicas (temperatura, precipitação pluviométrica, umidade relativa do ar) com os valores de OPG para ovos tipo Strongyloidea, bem como para determinação do grau de associação entre os valores de OPG e LPG.

Os coeficientes de correlação foram analisados segundo a tabela de RUGG, considerando-se também o erro-padrão em relação à correlação.

\footnotetext{
1 Caprinotec - Purina
} 


\section{Resultados e discussão}

Foram analisadas 250 amostras, obtendo-se um percentual de positividade de $82 \%$. Das amostras examinadas, $72,8 \%$ apresentavam ovos tipo Strongyloidea, $8,4 \%$ foram positivas para Moniezia sp., e $2 \%$ para Trichuris sp. (Tabela 1). O grau de infecção variou de leve a pesado, com predominância do grau moderado nas amostras positivas para ovos tipo Strongyloidea.

Tabela 1 - Valor médio e desvio-padrão mensais do número de ovos por grama de fezes (OPG) em caprinos criados no município de Paulista, PE no período de agosto de 1998 a julho de 1999

\begin{tabular}{ccccccc}
\hline MÉS/ANO & \multicolumn{2}{c}{ Strongyloidea } & \multicolumn{2}{c}{ Moniezia sp. } & \multicolumn{2}{c}{ Trichuris sp. } \\
\hline & Média & DP & Média & DP & Média & DP \\
$8 / 98$ & 475,00 & 465,57 & 35,71 & 119,30 & 0,00 & 0,00 \\
$9 / 98$ & 303,85 & 581,36 & 0,00 & 0,00 & 0,00 & 0,00 \\
$10 / 98$ & 214,29 & 528,07 & 26,79 & 72,63 & 8,93 & 38,62 \\
$11 / 98$ & 366,67 & 900,19 & 9,52 & 30,08 & 14,29 & 47,81 \\
$12 / 98$ & 235,00 & 797,55 & 25,00 & 55,01 & 0,00 & 0,00 \\
$1 / 99$ & 52,63 & 84,12 & 0,00 & 0,00 & 0,00 & 0,00 \\
$2 / 99$ & 404,76 & 299,96 & 11,90 & 38,42 & 4,76 & 21,82 \\
$3 / 99$ & 623,53 & 675,95 & 5,88 & 24,25 & 0,00 & 0,00 \\
$4 / 99$ & 1421,05 & 1003,09 & 0,00 & 0,00 & 0,00 & 0,00 \\
$5 / 99$ & 3015,00 & 2435,11 & 175,00 & 782,62 & 0,00 & 0,00 \\
$6 / 99$ & 3964,29 & 5000,86 & 14,29 & 53,45 & 0,00 & 0,00 \\
$7 / 99$ & 8376,47 & 7333,70 & 0,00 & 0,00 & 0,00 & 0,00 \\
\hline
\end{tabular}

DP - Desvio-padrão.

Maiores indices de amostras negativas concentraram-se nos meses de setembro, outubro, dezembro/1998 e janeiro/1999, os quais apresentaram, respectivamente, indices pluviométricos de $52,3 \mathrm{~mm}, 54,9 \mathrm{~mm}, 16,0 \mathrm{~mm}$ e $44,7 \mathrm{~mm}$, correspondendo ao período seco. Verificou-se um expressivo aumento do número de ovos tipo Strongyloidea por gramas de fezes no período chuvoso na área estudada, ocorrendo nos meses de abril, maio, junho, julho/1998 (Figura 1). Fato semelhante foi encontrado por Girão et al. (1978), no Piauí, onde encontraram aumento de OPG no período chuvoso e diminuição na época seca.

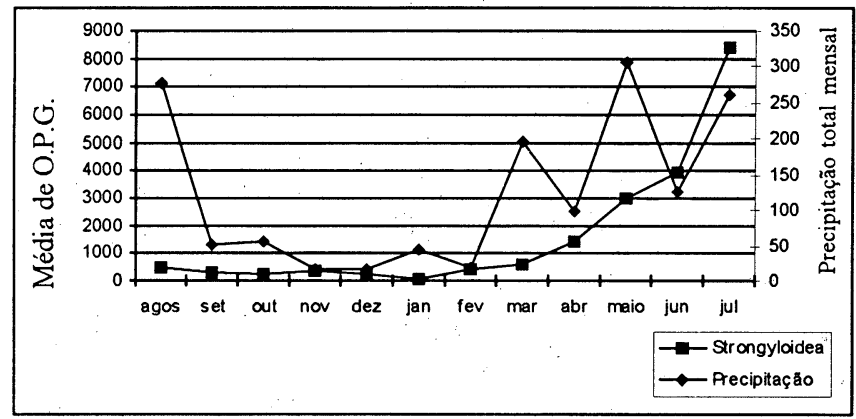

Figura 1 - Variação do número médio mensal de ovos tipo Strongyloidea por grama de fezes em caprinos no periodo de 8/98 a 7/99 em relação à precipitação total mensal no municipio de Paulista, PE
À identificação de larvas de terceiro estágio, os gêneros de helmintos encontrados foram Haemonchus sp. $(75,13 \%)$, Trichostrongylus sp. (24,32\%) e Oesophagostomum spp. $(0,54 \%)$, sendo estes gêneros registrados por Girão et al., (1992) no Piauí; e por Almeida et al., (1997), na Bahia e Cavalcante e Vieira (1992), Cavalcante e Lopes (1997), no Ceará.

À análise de correlação (Tabela 2), obteve-se correlação significativa do OPG com temperatura e precipitação pluviomé-trica, verificando-se que, nos meses em que a temperatura apresentou-se menos elevada, houve aumento do OPG; no entanto, nesses mesmos meses, foram observados também indices mais elevados de precipitação pluviométrica. Constatou-se uma correlação altamente significativa entre OPG e o número de larvas de Haemonchus sp. por grama de fezes, não havendo correlação entre o OPG e LPG para Trichostrongylus sp. Este fato vem reforçar a afirmação feita por Morales et al. (1998), de que o número de ovos eliminados nas fezes está relacionado com a espécie do parasito, sendo a alta profilicidade uma característica do Haemonchus sp.

O gênero Haemonchus ocorreu durante todo o ano, variando o número médio mensal de larvas por grama de fezes de 0,04 a 51,67 (Figura 2). Com relação ao gênero Trichostrongylus, observou-se variação de 0,00 a 1,34 larvas por grama de fezes (Figura 3). O número médio de larvas por grama de fezes para os gêneros Haemonchus e Trichostrongylus foi maior na estação chuvosa, como observado também por Charles (1995). Este autor afirma que a umidade é necessária ao desenvolvimento dos ovos e

Tabela 2 - Coeficiente de Correlação entre OPG e fatores climáticos e entre o OPG para ovos tipo Strongyloidea e LPG de Haemonchus sp. e Trichostrongylus sp. para caprinos criados no município de Paulista, PE

\begin{tabular}{cccccc}
\hline Variáveis & Temperatura & Precipitação & $\begin{array}{c}\text { Umidade } \\
\text { relativa }\end{array}$ & $\begin{array}{c}\text { LPG } \\
\text { (HAE) }\end{array}$ & $\begin{array}{c}\text { LPG } \\
\text { (TRIC) }\end{array}$ \\
\hline SDEA & $-0,6113$ & 0,5607 & 0,3593 & 0,8799 & 0,1588 \\
\hline SDEA - Strongy
\end{tabular}

SDEA - Strongyloidea.; HAE - Haemonchus sp.; TRIC - Trichostrongylus sp.

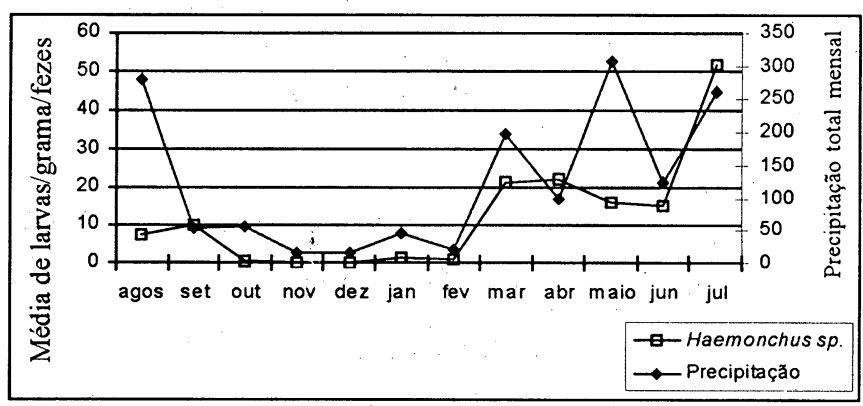

Figura 2 - Variação do número médio mensal de larvas de Haemonchus sp. por grama de fezes em caprinos no período de 8/98 a 7/99 em relação à precipitação total mensal no município de Paulista, PE 


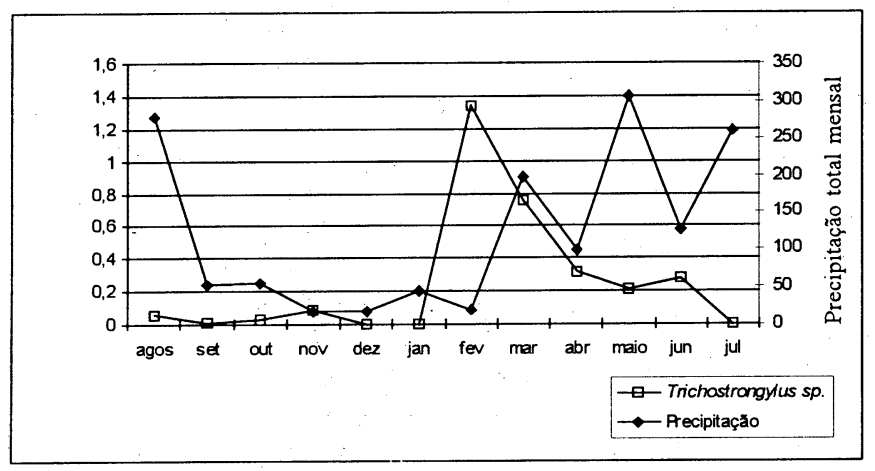

Figura 3 - Variação do número médio mensal de larvas de Trichostrongylus sp. por grama de fezes em caprinos no período de 8/98 a 7/99 em relação à precipitação total mensal no município de Paulista, PR

larvas nas pastagens, assim como para migração das larvas para a vegetação ao redor dos bolos fecais. Desta forma a infecção dos animais é favorecida, influenciando a carga parasitária e consequentemente a eliminação de ovos, refletindo-se no número de larvas. A influência dos fatores climáticos sobre a população de helmintos também foi estudada por Girão et al. (1997), na região de Inhamuns, onde observaram uma carga parasitária média de 113,65 helmintos, durante 0 período de seca, com índice pluviométrico de $1,3 \mathrm{~mm}, \mathrm{e}$, no período de chuvas, com índice pluviométrico de $549,4 \mathrm{~mm}$, uma carga parasitária média de 1035,75 helmintos.

\section{Referências}

ALMEIDA, M.A.O.;AYRES, M.C.C.; SANTOS, S.C.S. Freqüência de helmintos gastrintestinais em caprinos leiteiros criados no estado da Bahia: influência do sistema de criação e dos fatores etários e racial. In: SEMINÁRIO BRASILEIRO DE PARASITOLOGIAVETERINÁRIA, 10., 1997, Itapema - SC. Anais..., Itapema: Colégio Brasileiro de Parasitologia Veterinária, 1997, p. 228

BIANCHIN, I. Epidemiologia dos nematódeos gastrintestinais em bovinos de corte nos Cerrados e o controle estratégico no Brasil. In: PADILHA, T. (Ed.) Controle dos nematóideos gastrintestinais em ruminantes. Coronel Pacheco, 1996. p.113-156.

CASTRO, A . A cabra. 2. ed. Fortaleza: Imprensa Oficial, 1981. 376 p. CAVALCANTE, A.C.R.; LOPES, C.W.G. Parasitos gastrointestinais de caprinos leiteiros na microrregião homogênea de Sobral, Ceará. In: SEMINÁRIO BRASILEIRO DE PARASITOLOGIAVETERINÁRIA, 10., Itapema - SC., Anais... Itapema: Colégio Brasileiro de Parasitologia Veterinária, 1997, p. 230.

CAVALCANTE, A.C.R.; VIEIRA, L.S. Importância do pastejo misto (caprino com ovinos), na incidência de nematódeos gastrintestinais. In: CONGRESSO BRASILEIRO DE MEDICINA VETERINÁRIA., 22., 1992 Curitiba, Anais..., Paraná, p. 382, 1992.

CHARLES, T.P. Disponibilidade de larvas infectantes de nematóides gastrointestinais parasitas de ovinos deslanados no semi-árido pernambucano. Ciência Rural., v. 25, n. 3, p. 437-442,1995.

CHARLES, T.P. Seasonal prevalence of gastrointestinal nematodes of goats in Pernambuco state, Brazil, Veterinary Parasitology, n. 30, p. 335-343,1989.

COSTA, C.A.F. Helmintoses de caprinos e ovinos: estágio atual da pesquisa no Nordeste brasileiro. In: SEMINÁRIO BRASILEIRO DE PARASITOLOGIAVETERINÁRIA, n. 2, 1980, Fortaleza, Anais... Brasilia: EMBRAPA, 1980. p. 41-58.
Outro fator de influência na população de helmintos seria o tratamento anti-helmíntico (Vieira, 1999). Neste estudo, em particular, o fato de as vermifugações terem-se concentrado nos meses de menor precipitação pluviométrica, sendo reduzidas nos meses do período chuvoso, aliado às condições ambientais, deve também ser levado em consideração, em relação ao aumento do OPG verificado nesta época durante o período experimental.

A infecção por Oesophagostomum spp. foi observada apenas em um mês (novembro/1998). Isto pode indicar ocorrência esporádica deste gênero em caprinos na região estudada, semelhante às observações de Grisi (1975), também em caprinos, na Bahia.

\section{Conclusão}

Nas condições em que se realizou o estudo, chega-se à seguinte conclusão:

Ocorre infecção por parasitos gastrintestinais em níveis consideráveis no rebanho estudado, principalmente por helmintos dos gêneros Haemonchus sp., Trichostrongylus spp., e esporadicamente infecção por Trichuris sp. e Oesophagostomum spp.; sendo a precipitação pluviométrica o fator climático de maior influência na variação dos índices de infecção. A infecção por cestóides do gênero Moniezia spp. ocorre em níveis reduzidos.

COSTA, H.M.A.; FREITAS, M.G; GUIMARĀES, M.P. Prevalência e intensidade de infecção por helmintos de bovinos procedentes da área de Três Corações. Arquivo da Escola de Veterinária da Universidade Federal de Minas Gerais, n. 22, p. 95-101, 1970.

COSTA, J.O.; GUIMARÃES, M.P. Helmintos parasitos de bezerros procedentes da bacia leiteira de Caciolândia - Brasil. Arquivo da Escola de Veterinária da Universidade Federal de Minas Gerais, v. 25, n. 2, p. 111-116, 1973.

COSTA, J.O.; GUIMARÃES, M.P. Helmintos parasitos de bezerros procedentes da bacia leiteira de Ibiá - MG Arquivo da Escola de Veterinánia da Universidade Federal de Minas Gerais, n. 23, p. 221-228, 1971.

COSTA, C.A.F. Epidemiologia das helmintoses caprinas. In: SEMANA BRASILEIRA DO CAPRINO, 2., 1978, Sobral, CE, Anais... Sobral: EMBRAPA, 1982, p. 85-97.

CUNHA, T.S. The animals food resource for man. In: Int. Conf. Goat: prod. and disease. v. 8, p. 1-6, 1982.

GIRÃO, M.D. et al. Prevalência e intensidade de helmintos gastrintestinais de ovinos e caprinos da Região dos Inhamuns, Ceará. In: SEMINÁRIO BRASILEIRO DE PARASITOLOGIAVETERINÁRIA, 10., 1997. Anais... Itapema: Colégio Brasileiro de Parasitologia Veterinária, 1997, p. 214. GIRÃO, E.S., MEDEIROS, L.P.; GIRĀO, R.N. Ocorrência e distribuição estacional de helmintos gastrointestinais de caprinos no município de teresina, Piaui. Ciência Rural, v. 22, n. 2, p. 197-202, 1992.

GIRÃO, R.N.; GIRÃO, E.S.; MEDEIROS, L.P. Incidências de helmintos gastrointestinais de caprinos. Microrregião de Campo Maior e Valença do Piauí. Teresina: EMBRAPA, 1978, 7 p.(Comunicado técnico, n.8).

GORDON, H. Mcl ; WHITLOCK, H. V. A New technique for counting nematoda eggs in sheep faeces. Journal of the Council for Scientific and Industrial Research, v. 12, n. 1, p. 50-52, 1939.

GRISI, L. Incidência de helmintos em Capra Hircus procedentes do estado da Bahia. Revista Brasileira de Biologia, v. 35, n. 1, p. 101$108,1975$. 
I.B.G.E. Produção da Pecuária Municipal, Brasil; Instituto Brasileiro de Geografia e Estatística, 1998.

MORALES, $G$. et al. Relación entre la carga parasitaria, las especies del orden Strongylida presentes y el número de huevos en heces de caprino naturalmente infectados. Veterinaria Tropical. v. 23, n. 2, p. 101-107, 1998

PINHEIRO,A. C. Verminose ovina. A Hora Veterinária, ano 2, n. 12, 1983. ROBERTS, F. H. S.; O'SULLIVAN, J. P. Methods for egg counts and larval cultures for Strongyles infesting the gastrointestinal tract of cattle. Australian Agricultural Research, v. 1, p. 99-102, 1950.

SANTIAGO, M.A. M. Aspectos da biologia dos nematóideos: Delineamento de eclosão de ecossistema para estudos epidemiológicos. In
SEMINÁRIO NACIONAL SOBRE PARASITOSES DOS BOVINOS, 1. 1979, MS. Anais ... Campo Grande: EMBRAPA, 1979. P. 25-30.

SANTOS, S.M. Prejuizos econômicos determinados pelos parasitas Balde Branco, v. 18, p. 18-21,1982.

UENO, H.; GONÇALVES, P. C. Manual para diagnóstico das helmintoses de ruminantes. 4. ed. Tokyo: Japan Internacional CorporationAgency, 1998. $143 \mathrm{p}$.

VIEIRA, L. S. Epidemiologia e controle da nematodiose gastrintestinal dos caprinos. In: CONGRESSO PERNAMBUCANO DE MEDICINA VETERINÁRIA, 4., 1999, Recife. Anais... Recife: Sociedade Pernambucana de Medicina Veterinária, 1999. p.123. 